\title{
DESIGN \& CHARACTERIZATION OF A MEMS THERMAL SWITCH
}

\author{
J.H. Cho ${ }^{1}$, C.D. Richards ${ }^{1}$, J. Jiao ${ }^{2}$, D.F. Bahr ${ }^{1}$, and R.F. Richards ${ }^{1}$ \\ ${ }^{1}$ School of Mechanical and Materials Engineering Washington State University, Pullman WA, USA \\ ${ }^{2}$ Dept. of Physics, Portland State University, Portland OR USA
}

\begin{abstract}
In this paper we present the design, fabrication, and characterization of a MEMS thermal switch. Three switch designs are considered: one in which the conductive material is in the form of liquid-metal micro droplets, one in which the conductive material consists of aligned carbon nanotube arrays, and one in which solid silicon contacts are used. The ratio of thermal resistance in the on and off conditions is used to characterize performance. Based on these measurements the switch incorporating liquid-metal droplets is superior to the other two switches. The liquid-metal switch has a thermal resistance off to on ratio of 168 and can provide heating rates of $905^{\circ} \mathrm{C} / \mathrm{s}$.
\end{abstract}

\section{INTRODUCTION}

The ability to control heat transfer on small time and length scales could improve the performance of many micro devices; such as, thermoelectric micro-coolers, DNA amplification microchips, and some micro heat engines. First, it has been demonstrated that the performance of thermoelectric coolers could be nearly doubled if operated in a transient or pulsed mode $[1,2]$. Second, DNA amplification via PCR requires precise temperature control. The use of small sample sizes $(\sim 1 \mu \mathrm{l})$ and microfluidic devices has already dramatically reduced both the size and power needs of the equipment needed and the time required for DNA amplification [3]. Third, using waste heat to do mechanical work on the micro scale can be accomplished by controlling the flow of waste heat. For example, our group at Washington State University has demonstrated a MEMS-based micro heat engine that can harvest low-temperature heat to do mechanical work and produce electrical power [4].

To be able to control heat transfer to thermoelectric coolers, micromachined PCR devices and micro heat engines a type of thermal switch or thermal valve is required. Such a thermal switch would be able to change its effective thermal conductivity in order to turn heat transfer on and off. A figure of merit for such a device is the ratio of thermal contact resistance in the off and on positions, i.e., $R_{\text {off }} / R_{\text {on }}$ of the switch. As shown in Fig. 1, a high thermal resistance, $R_{\text {off, }}$ is required in the off position, so that the switch does not 'leak' heat. This off resistance is governed primarily by

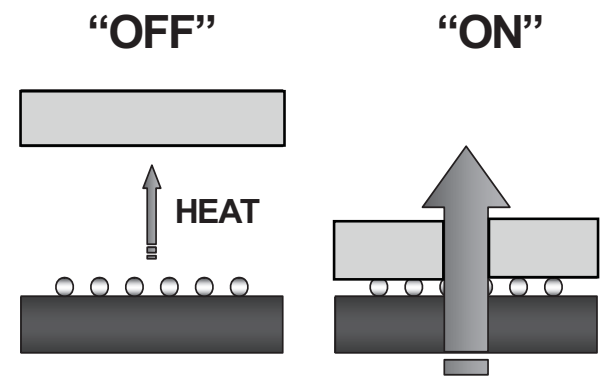

Figure 1. Thermal switch concept. the thermal properties of the gap. A very low thermal resistance, $R_{o n}$, is required in the on position, so that the heat transfer may be maximized. This on resistance depends primarily on the properties of the contacts used in the switch material.

\section{EXPERIMENTAL METHODS}

In this paper we address the effect of the thermal contact material. The thermal resistance of thermal switches with patterned contacts of either liquid-metal micro droplets or vertically aligned carbon nanotubes are compared to the thermal resistance of thermal switches with solid silicon contacts. The speed of switching is assessed by measuring the transient temperature history of the switch under dynamic operation.

We have used two types of patterned arrays of contacts for thermal switches: liquid-metal micro droplets and vertically aligned carbon nanotubes (VACNT). The liquid-metal droplet arrays consist of a $40 \times 40$ grid of 1600 mercury, 30- $\mu \mathrm{m}$ diameter, micro droplets deposited via selective vapor deposition. The vertically aligned carbon nanotube arrays consist of bundles of multiwall carbon nanotubes patterned in the same grid and with the same diameter as the micro droplets. Arrays of liquid-metal micro droplets, Fig. 2, and arrays of VACNT's, Fig.3, are fabricated on a silicon die. A second silicon die is used to make and break contact

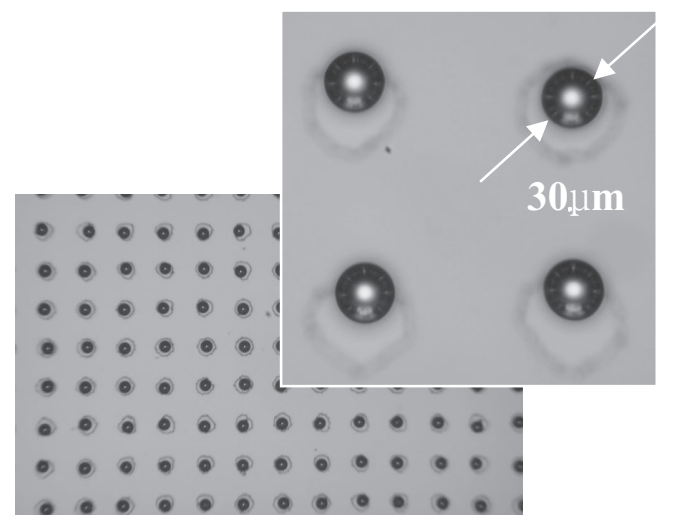

Figure 2. Liquid-metal micro droplets.

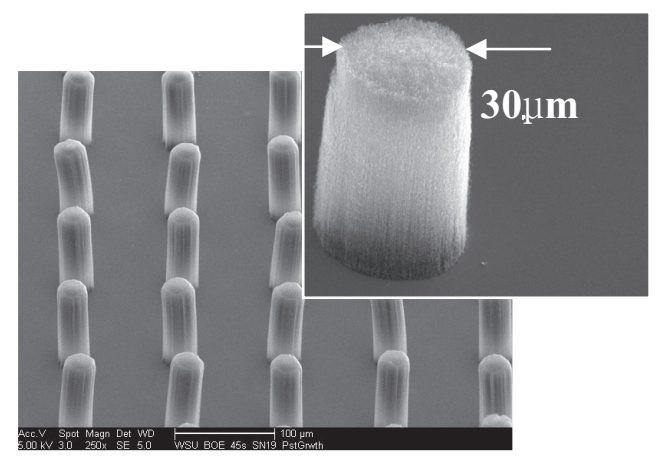

Figure 3. Vertically aligned carbon nanotube arrays. 
with the micro-droplet or VACNT array. When the die is mechanically actuated to make contact, squeezing the thermal contact array between them, the thermal switch is in its "on" state. When the die breaks contact, creating a gas gap between the die, the thermal switch is in its "off" state.

Liquid-metal micro-droplet arrays are fabricated by preferentially condensing mercury vapor on gold targets patterned on the silicon die. First, wet oxidation is used to grow $100-\mathrm{nm}$ thick silicon dioxide layers on both sides of a (100), 3-inch diameter silicon wafer. On the back side of the wafer, a 12-nm layer of titanium and a $175-\mathrm{nm}$ thick layer of platinum are deposited using DC magnetron sputtering. Platinum Resistance Thermometers (PRT's) and heaters are fabricated by defining serpentine traces using a platinum-liftoff method. These PRT's and heaters are used to characterize the thermal switch. The front side of the wafer is sputtered with a 5-nm adhesion layer of titanium/tungsten followed by a $300-\mathrm{nm}$ layer of gold. The gold is then patterned via photolithography to produce a $4 \mathrm{~mm}$ square, 40 x 40 pattern of $160020-\mu \mathrm{m}$ diameter circular gold targets. The gold targets are subsequently covered with a $1.5-\mu \mathrm{m}$ thick layer of photoresist. The photoresist is then etched around the gold targets as shown in Fig. 2. The $40 \times 40$ grids of circular gold targets on the front of the wafer are aligned directly opposite the resistance heaters and PRT's defined on the back of the wafer [5].

CNT growth is accomplished via a chemical vapor deposition (CVD) method. An iron nitrate sol gel catalyst is spun onto a wafer followed by photoresist and then patterned. A (100) silicon wafer is prepared for sol gel spin coating by etching in buffered oxide etchant (BOE) to remove the native oxide layer. Next, the sol gel solution is spun onto the silicon wafer. After cleaning, the iron containing silicon dioxide catalyst is patterned. A traditional photolithographic process is used to pattern the sol gel catalyst. Next, the wafer is exposed to UV light masked with a 1600 dot array identical to that used for the mercury droplet array. The photoresist is developed, and the wafer rinsed and dried. The wafer is then etched in BOE. After etching the photoresist is stripped, the wafer is cleaned, and then diced.

Multi-walled carbon nanotube growth is accomplished through the use of the CVD reactor. The sol gel patterned dies are placed in a ceramic boat in the center of a quartz tube, which is in turn inserted into a horizontal tube furnace. The tube is evacuated to 120 milliTorr and a three stage process follows: catalyst calcinations, catalyst activation, and MWCNT growth with an admixture of $\mathrm{H}_{2}$ and $\mathrm{C}_{2} \mathrm{H}_{2}$. After the growth period, the tube is evacuated and allowed to cool to room temperature [7].

The MEMS thermal switches are first characterized under steady state conditions. The apparatus is shown in Fig 4. The test facility enables the control of the applied force squeezing the contacts (silicon surfaces, liquid-metal micro-droplet arrays or

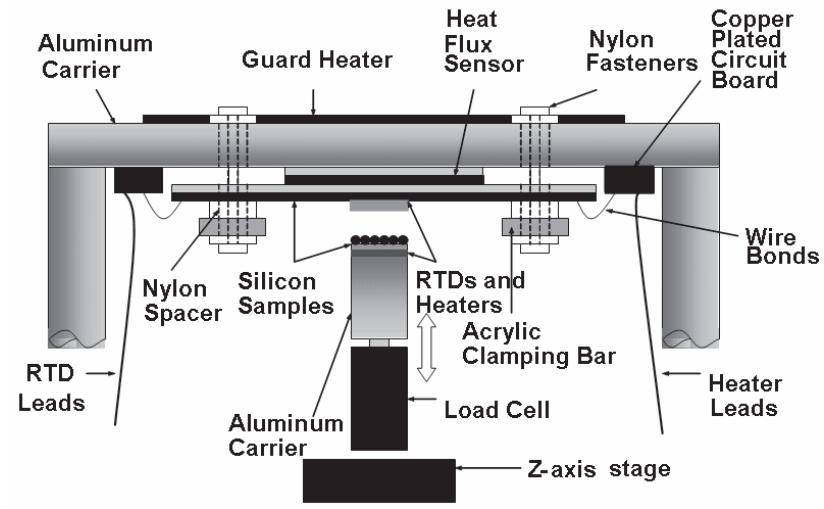

Figure 4. Schematic of steady state heat transfer experiments.
VACNT arrays) when the thermal switch is on, the thickness of the gas gap when the thermal switch is off, and the gas pressure in the gap. The silicon die, on which the thermal switch contacts are fabricated, the contact die, makes up the bottom half of the thermal switch. That contact die is mounted on the aluminum carrier shown in Fig. 4. A guard-heated calorimeter, used to measure heat transfer across the thermal switch, makes up the top half of the thermal switch [6]. The guard-heated calorimeter, also shown in Fig 4, consists of a silicon heater die, a heat flux sensor and a guard heater, all mounted on a rigid aluminum plate. In order to make thermal resistance measurements, the heat flux sensor is zeroed by controlling power delivered to the guard heater. Under this condition, all the electrical power dissipated in the silicon heater die is transferred as heat down through the thermal switch to the bottom contact die. The heat transfer rate across the switch is thus equal to the input power to the silicon heater die and is determined from the current supplied and the voltage drop across the heater. The temperature difference across the thermal switch is measured with PRT's micromachined on the top silicon heater die and the bottom contact die. The thermal resistance across the thermal switch is taken to be the ratio of the temperature difference across the thermal switch over the heat transfer rate across the thermal switch.

To characterize the dynamic behavior of the switch a radial heat flux sensor, shown in Fig. 5, has been designed and micromachined in silicon. The heat flux sensor consists of a $2-\mu \mathrm{m}$ thick, 1.2-mm square membrane etched into a silicon wafer. Two concentric, annular platinum resistance thermometers (100-nm thick) are fabricated on the membrane at radii of $4.25-\mathrm{mm}$, and 5.5- $\mathrm{mm}$ respectively. A third PRT is defined in a square pattern at the center of the membrane. Measurements are made by periodically bringing the thermal switch contact die into and out of contact with the center of the membrane heat flux sensor. As the thermal switch contacts make and break contact with the membrane, the temperature of the contact die, $T_{1}$, the temperature at the center of the membrane heat flux sensor, $T_{2}$, and the temperatures at the two concentric annular PRT's: $T_{3}$ at a radius of $r_{3}=4.25-\mathrm{mm}$, and $T_{4}$ at a radius of $r_{4}=5.5-\mathrm{mm}$ are recorded. The heat transfer rate across the thermal switch can be determined from the temperature difference across the two annular PRT's [8]:

$$
q=\frac{2 \pi L k\left(T_{3}-T_{4}\right)}{\ln \left(r_{4} / r_{3}\right)}
$$

where $L$ is the membrane thickness of the radial heat flux sensor, $k$ is the thermal conductivity of the silicon membrane, and $r_{3}$ and $r_{4}$ are the respective radii of the temperature measurements.

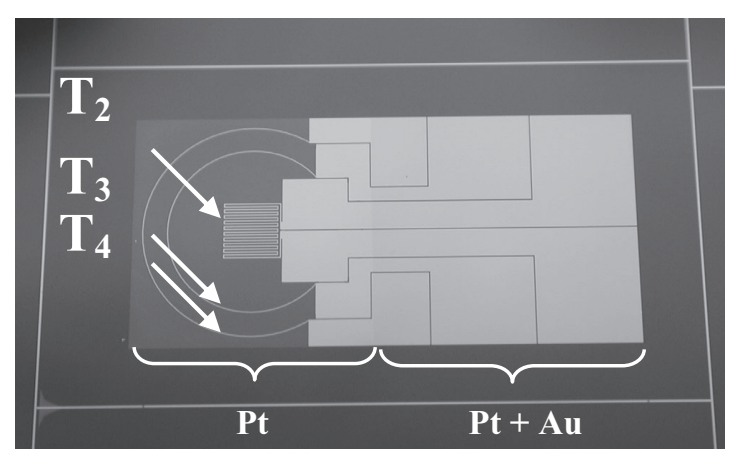

Figure 5. Radial heat flux sensor. 
The temperature difference across the thermal switch is the difference between the temperature of the contact die, $T_{1}$, and the temperature at the center of the membrane heat flux sensor, $T_{2}$. The thermal resistance across the thermal switch is once again taken to be the ratio of the temperature difference across the thermal switch over the heat transfer rate across the thermal switch.

The movement of the contact die into and out of contact with the membrane heat flux sensor is controlled by a piezo stack actuator. The displacement of the thermal switch contact die is measured with laser vibrometer. The load applied as the contact die comes into contact with the membrane heat flux sensor is measured with a $S$ type load cell which is attached between the piezo stack actuator and the thermal switch contact die.

\section{RESULTS}

Three switch conductor materials were tested, polished silicon surfaces, Hg micro droplets, and VACNT's. All measurements for steady state conditions were obtained in a reduced pressure atmosphere of air at 0.45 Torr. The results for the three switches in the off state are shown in Fig. 6. The thermal resistance of the "off" condition is characterized with respect to the gap thickness between the top and bottom dies. In the off position, the three switches have essentially the same thermal resistance. The thermal resistances for the three contact dies are seen to increase from 130 ${ }^{\circ} \mathrm{C} / \mathrm{W}$ when the gas gap thickness is 20 microns, to $150{ }^{\circ} \mathrm{C} / \mathrm{W}$ when the gas gap thickness is 100 microns, close to the mean free path length of $126-\mu \mathrm{m}$ for these conditions.

The thermal resistance of the "on" condition switch is characterized as a function of the applied load squeezing the switch closed. These data are shown in Fig. 7. For the closed, switch "on", condition, the thermal resistance of the contacts controls the heat transfer. For the $\mathrm{Hg}$ micro-droplet array the thermal resistance varies from $1.2{ }^{\circ} \mathrm{C} / \mathrm{W}$ at an applied load of $0.1 \mathrm{~N}$ to 0.6 ${ }^{\circ} \mathrm{C} / \mathrm{W}$ at $1 \mathrm{~N}$. The thermal resistance of the VACNT array varies from $32{ }^{\circ} \mathrm{C} / \mathrm{W}$ at an applied load of $0.1 \mathrm{~N}$ to $15^{\circ} \mathrm{C} / \mathrm{W}$ at $1 \mathrm{~N}$. The silicon die contacts have a thermal resistance that varies for varies from $49{ }^{\circ} \mathrm{C} / \mathrm{W}$ at an applied load of $0.1 \mathrm{~N}$ to $34{ }^{\circ} \mathrm{C} / \mathrm{W}$ at $1 \mathrm{~N}$. In the on position, the $\mathrm{Hg}$ micro droplets have the lowest thermal resistance. The relatively high values of the VACNT and silicon switches are due to contact resistance. The mechanisms for the contact resistance in the two switches are different. In the silicon to silicon switch the presence of asperities, contaminants, or other imperfections in the two mating surfaces can lead to a condition in which the actual contact area is severely reduced. Heat transfer then occurs primarily by conduction through the gas gap. In the case of the VACNT structures the contact resistance is governed by much more complex interface phenomena that are currently an

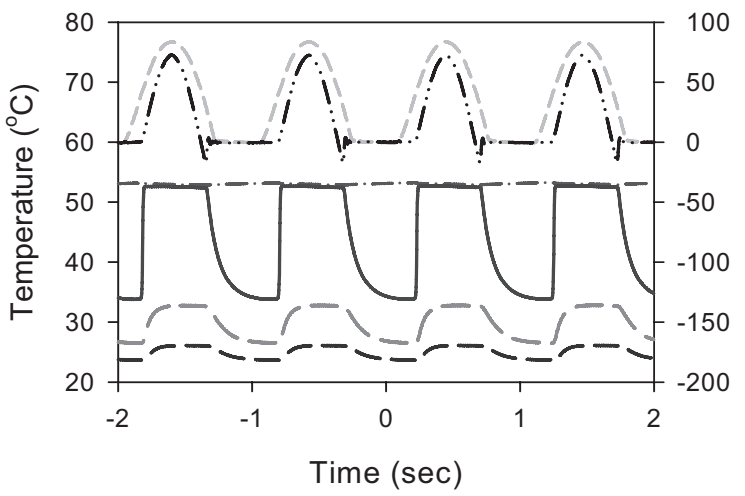

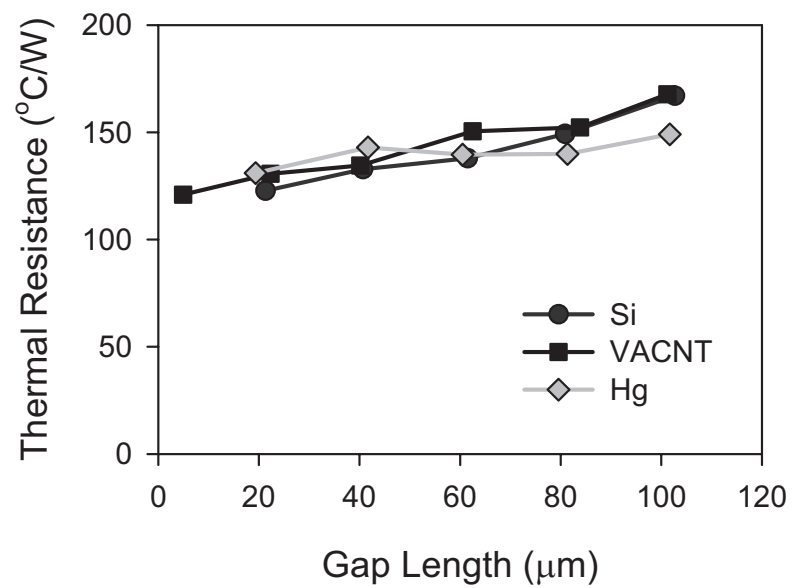

Figure 6. Thermal resistance for switch off.

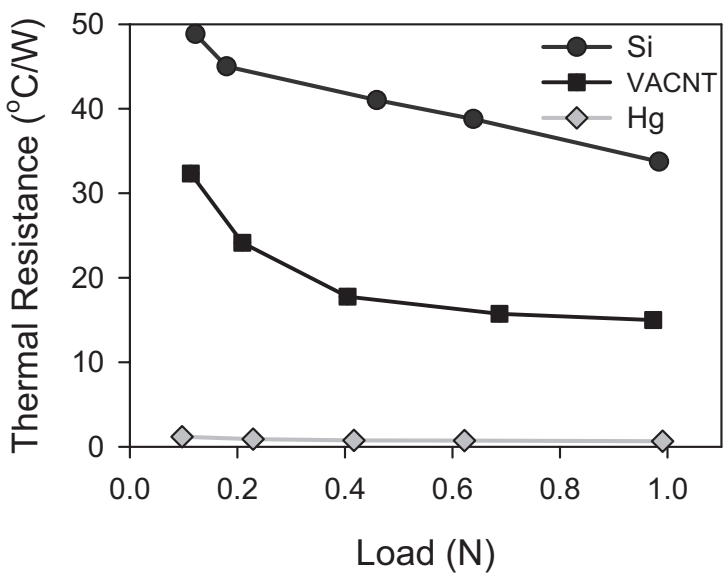

Figure 7. Thermal resistance for switch on.

active area of research.

The performance of the thermal switches may be characterized by the nondimensional ratio of the "off" state thermal resistance to the "on" state thermal resistance: $R_{\text {off }} / R_{o n}$. For the $\mathrm{Hg}$ micro droplet switch the thermal resistance ratio, $R_{\text {off }} / R_{o n}$, is 168 . The value of $R_{\text {off }} / R_{\text {on }}$ for the VACNT switch is 6.8 and for the siliconsilicon switch is 3.4. The $\mathrm{Hg}$ micro-droplet switch is clearly superior.

The dynamic response of a liquid-metal switch is shown in Fig. 8. The test was performed in a reduced pressure atmosphere of air at 0.1 Torr. The time history of four temperatures are

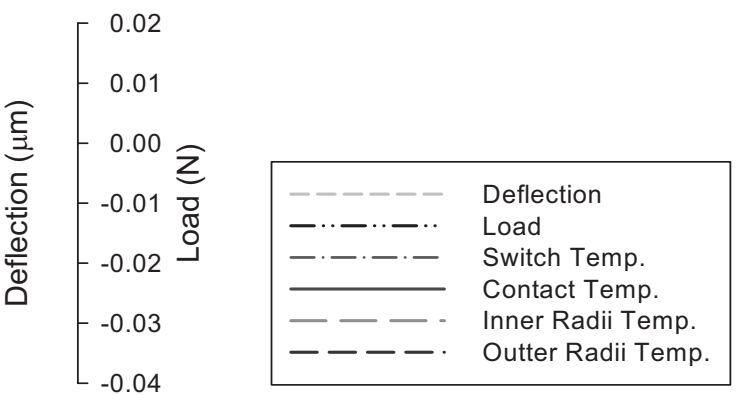

Figure 8. Dynamic behavior of thermal switch. 
shown: the switch temperature $\left(T_{1}\right)$ of the micro-droplet array die, the center contact temperature $\left(T_{2}\right)$, along with the radial heat flux sensor's inner and outer radii temperatures $\left(T_{3}\right.$ and $\left.T_{4}\right)$. The switch deflection and applied load are also shown. The location of $T_{2}, T_{3}$, and $T_{4}$ are shown in Fig 5 .

As the lower die, with the liquid-metal micro-droplet arrays, contacts the center of the heat flux sensor (on the top die), the switch turns "on" and the temperature increases. This occurs in 0.02 seconds as seen in Fig. 9. The figure shows a 0.02 second window of one of the contact events depicted in Fig. 8. The switch turns "on" at $\mathrm{t}=0.22$ seconds and the temperature increases from $34.4{ }^{\circ} \mathrm{C}$ to $52.5^{\circ} \mathrm{C}$ over a span of 0.02 seconds. The heating rate is thus $905{ }^{\circ} \mathrm{C} / \mathrm{sec}$. The heat transferred is determined from the radial heat flux sensor measurements of $T_{3}$ and $T_{4}$ when the switch is on. In this case $T_{3}$ and $T_{4}$ are $32.8{ }^{\circ} \mathrm{C}$ and $26.1{ }^{\circ} \mathrm{C}$ respectively which yields a heat transfer rate of $51 \mathrm{~mW}$ from Eqn (1).

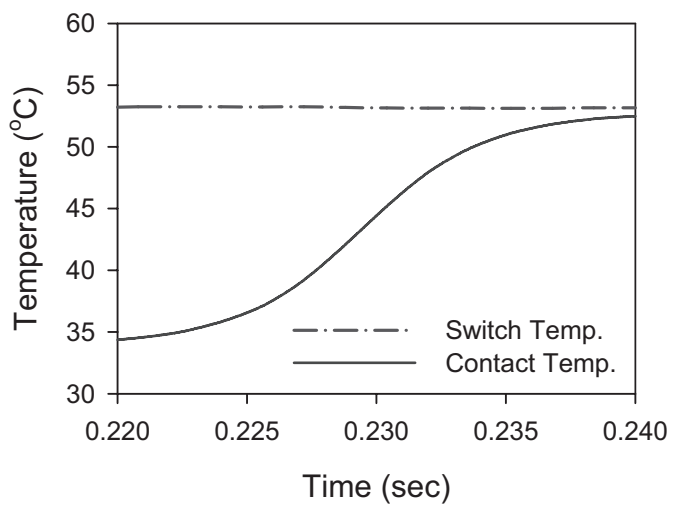

Figure 9. The switching speed of the thermal switch.

\section{SUMMARY}

In this work the design, fabrication and testing of a thermal switch are presented. Three switch conductor materials were tested: polished silicon surfaces, arrays of $\mathrm{Hg}$ micro droplets, and arrays of VACNT's. The switch performance was characterized by the ratio of thermal resistance in the switch off to on position. The on state thermal resistances of the VACNT switch and the silicon contact switch showed relatively high values of contact resistance. The liquid-metal micro-droplet switch showed a much smaller contact resistance at the same applied load. As a result, the liquid-metal micro-droplet switch had the best performance of the three switches tested with an off to on thermal resistance ratio of 168. The switching speed of the $\mathrm{Hg}$ micro-droplet switch was shown to be on the order of 0.02 seconds, realizing heating rates of $905^{\circ} \mathrm{C} / \mathrm{sec}$.

\section{REFERENCES}

[1] A. Miner, A. Majumdar and U. Ghoshal, "Thermomechanical refrigeration based on transient thermoelectric effects," Applied Physics Letters, 75, 1176-1178, (1999)

[2] G. J. Snyder, J-P. Fleurial, T. Caillat, R. Yang and G.Chen, "Supercooling of Peltier cooler using a current pulse," Journal of Applied Physics, 92, 1564-1569, (2002)
[3] C.T. Wittwer, G.C. Fillmore and D.J. Garling, "Minimizing the time required for DNA amplification by efficient heat transfer to small samples," Anal. Biochem., 186, 328-331, (1990)

[4] S. Whalen, M. Thompson, D. Bahr, C. Richards and R. Richards, "Design fabrication and testing of the P3 micro heat engine" Sensors \& Actuators: A. Physical, 104, 200-208 (2003).

[5] J.H. Cho, T. Wiser, C.D. Richards, D.F. Bahr and R.F. Richards, "Fabrication and Characterization of a Thermal Switch," Sensors and Actuators, in press.

[6] T. Wiser, "Steady State Heat Transfer Characterization of a Liquid Metal Thermal Switch," MS Thesis, WSU, 2005

[7] C. M. McCarter, D.F. Bahr, R.F. Richards, C.D. Richards, D. McClain, and J. Jiao, "Integration of Carbon Nanotubes with MEMS through Standard Photolithographic Techniques, MS\&T 2005, Nanomaterials Symposia, Pittsburgh, PA, September, 2005.

[8] Frank P. Incropera and David P. DeWitt, Introduction to Heat Transfer, Fourth Edition, John Wiley \& Sons, Inc., 104-107, (2002). 